\title{
Mitochondrial Function in Pluripotent Stem Cells and Cellular Reprogramming
}

\author{
Raul Bukowiecki ${ }^{a}$ James Adjaye ${ }^{\mathrm{b}, \mathrm{c}}$ Alessandro Prigione ${ }^{\mathrm{a}}$ \\ ${ }^{a}$ Max Delbrueck Center for Molecular Medicine (MDC), ${ }^{b}$ Department of Vertebrate Genomics, Molecular \\ Embryology and Aging Group, Max Planck Institute for Molecular Genetics, Berlin, and ' Institute for Stem \\ Cell Research and Regenerative Medicine, Heinrich Heine University, Dusseldorf, Germany
}

\author{
Key Words \\ Mitochondria $\cdot$ Metabolism $\cdot$ Reprogramming $\cdot$ iPS cells . \\ mtDNA $\cdot$ Stem cells
}

\begin{abstract}
Mitochondria are organelles playing pivotal roles in a range of diverse cellular functions, from energy generation to redox homeostasis and apoptosis regulation. Their loss of functionality may indeed contribute to the development of aging and age-related neurodegenerative disorders. Recently, mitochondria have been shown to exhibit peculiar features in pluripotent stem cells (PSCs). Moreover, an extensive restructuring of mitochondria has been observed during the process of cellular reprogramming, i.e. the conversion of somatic cells into induced pluripotent stem cells (iPSCs). These transformation events impact mitochondrial number, morphology, activity, cellular metabolism, and mtDNA integrity. PSCs retain the capability to self-renew indefinitely and to give rise to virtually any cell type of the body and thus hold great promise in medical research. Understanding the mitochondrial properties of PSCs, and how to modulate them, may thus help to shed light on the features of stemness and possibly increase our knowledge on cellular identity and differentiation pathways. Here, we review these recent findings and discuss their implications in the context of stem cell biology, aging research, and regenerative medicine.
\end{abstract}

\section{KARGER}

E-Mail karger@karger.com

www.karger.com/ger

\section{Pluripotent Stem Cells and Cellular Reprogramming}

Stem cells are endowed with two unique features: indefinite propagation (self-renewal) and the capability to generate a diverse range of specialized cell types through differentiation (potency). The degree of potency defines the stem cell identity. Adult (or somatic) stem cells, like human mesenchymal stem cells (MSCs) and hematopoietic stem cells (HSCs), which can be found in developed organisms, are multipotent, since their differentiation is restricted to specific lineages. On the other hand, pluripotent stem cells (PSCs), i.e. embryonic stem cells (ESCs) and induced pluripotent stem cells (iPSCs), retain the ability to differentiate into any cell type within the three embryonic germ layers.

ESCs were first isolated from the inner cell mass of the mouse blastocyst by Martin Evans in 1981, and later in humans, by James Thomson in 1998. Since then, the stem cell field has evolved immensely and considerable advances have been made to define the cell properties associated with stemness and the molecular mechanisms and pathways involved in specific differentiation routes. Nonetheless, in the context of regenerative medicine, work with human ESCs (hESCs) raises concerns related to their derivation from human blastocysts and to the potential risks of immunogenic reactions upon transplantation.

One way to circumvent these issues would be the generation of genetically equivalent stem cells, directly 
obtained from the patient's own cells (isogenic). Indeed, all cells of multicellular organisms possess the same genetic code but are functionally heterogeneous, due to their distinct epigenetic status. Thus, it may be conceivable that, through the modulation of their epigenetic profile, somatic cells may be reverted to stem cell-like cells capable of acquiring the same properties inherent to ESCs.

The first demonstration of this remarkable conversion was obtained with somatic cell nuclear transfer by John Gurdon in the late 1950s. His work established that differentiated cells are capable of reactivating genes that have become functionally inactive during development. Unfortunately, however, somatic cell nuclear transfer has been quite unsuccessful in humans and it may encounter similar ethical difficulties as hESC-based medicine, as it would also require the use of unfertilized human oocytes.

A second route to revert somatic cells to pluripotency was demonstrated by Shinya Yamanka in 2006 [1]. This innovative technique, called cellular reprogramming, is achieved by introducing ESC-specific transcription factors into somatic cells, which subsequently acquire ESClike properties. These somatic cell-derived PSCs, known as induced iPSCs, appear almost indistinguishable from ESCs in terms of gene expression, morphology, cell-cycle structure, epigenetic signature, and developmental potential. These features put forward patient-derived iPSCs as one of the most promising tools for regenerative medicine applications (see box 1).

Recently, the plasticity of the cellular epigenetic state was further proven by the demonstration that somatic cells can be converted into different somatic cells, a process now known as transdifferentiation or direct reprogramming. With this approach, fibroblasts can be directly turned into hepatocytes, cardiomyocytes, or specialized neurons, bypassing the iPSC state [2].

PSCs and cellular reprogramming appear as highly relevant instruments for investigating the biological aspects of cellular identity and to develop innovative approaches to study and possibly cure complex human disorders. Nonetheless, the mitochondrial properties of stem cells and reprogrammed cells have only recently started to be investigated. Here, we review this recent mitochondrial research with a specific focus on human PSCs. We use fibroblasts as exemplary somatic cells since most of the studies were conducted on these cells. However, it may be possible that different somatic cells may exhibit unique mitochondrial and metabolic characteristics.

Mitochondrial Function in Pluripotent

Stem Cells and Cellular Reprogramming
Box 1. iPSCs and their value for clinical research

After only 6 years' time, the ground-breaking work of Shinya Yamanaka [10] acquired world-wide recognition when he was awarded together with John Gurdon the Nobel Prize for Physiology or Medicine in 2012. The discovery that four transcription factors (OCT4, SOX2, KLF4, and c-MYC) are sufficient to reprogram differentiated somatic cells into ESC-like cells, designated as iPSCs, shifted the paradigm of unidirectional cellular development and cell fate. The idea of reprogramming influenced many areas of science, from developmental biology to medical research [18]. By enabling the derivation of unlimited patient-specific differentiated cells, the technology brought two major breakthroughs. First, the possibility to employ iPSCs for cellular replacement therapies, circumventing rejection complications through autologous transplantation. Second, the generation of human 'disease in a dish' cellular platforms, an innovative tool for modeling complex disorders. This appears particularly relevant for pathological conditions affecting tissues that are notoriously difficult to sample, e.g. components of the central nervous system. Reprogramming-derived patient-specific neurons are now being employed to advance the knowledge of disease-associated mechanisms and may eventually help the identification of novel therapeutic strategies for debilitating neurological disorders.

\section{Mitochondrial Features of Pluripotent Stem Cells}

Mitochondria are essential organelles present in all nucleated cells whose main function is the generation of cellular ATP by oxidative phosphorylation (OXPHOS), encompassing the electron transport respiratory chain (ETC, complexes I-IV) and the ATP synthase (complex $\mathrm{V})$. Mitochondria are also involved in a number of cellular pathways, including calcium homeostasis, apoptosis, heme biosynthesis, and cell signaling.

The subcellular content and distribution of mitochondria is vastly dynamic, as cellular polarity is considerably demonstrated by variations in mitochondrial number and configuration. The organelle turnover is facilitated by selective autophagy - or mitophagy - which degrades defective mitochondria via the lysosomal machinery. The enormous plasticity of the mitochondrial networks throughout the cell is maintained by constant balance between the two processes of organelle fusion and fission.

Structural analyses of mitochondria within undifferentiated human and mouse ESCs identified few organelles characterized by poorly developed cristae and perinuclear localization $[3,4]$. Accordingly, a low amount of mitochondrial DNA (mtDNA) copies have been detected in undifferentiated ESCs, while this number increases upon differentiation together with the level of mitochon- 

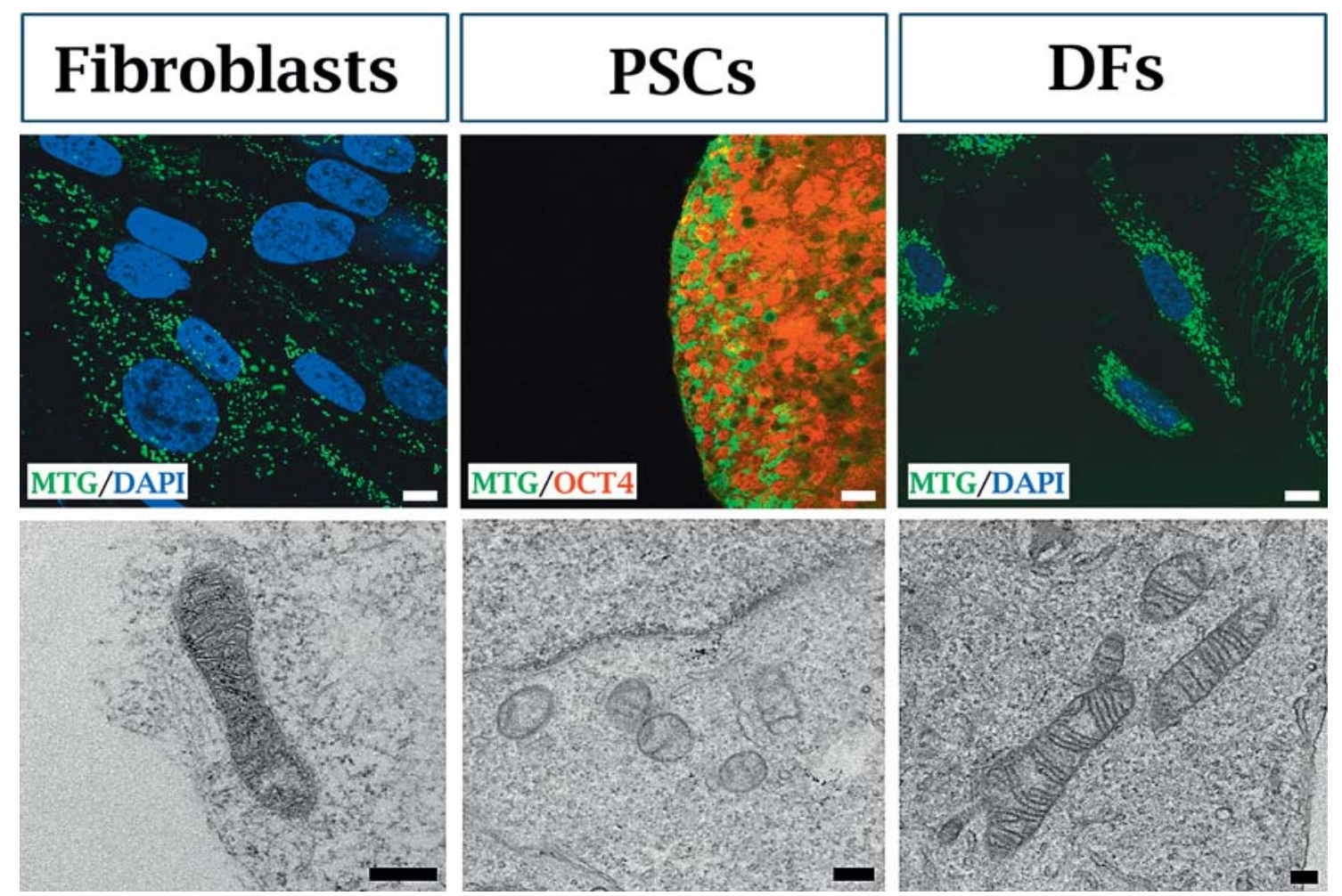

Fig. 1. Dynamics of mitochondrial morphology during reprogramming and differentiation. Somatic fibroblasts contain filamentous networks of respiratory-active mitochondria. Within PSCs, the mitochondrial morphology exhibits a spherical, condensed, immature-like structure. Upon differentiation into derived fibroblasts (DFs), mitochondria undergo structural maturation and acquire

drial maturation [5]. Hence, relatively underdeveloped mitochondrial network and low mitochondrial activity appear as features of 'stemness', as also confirmed in vivo in spermatogonia, inner cell mass, and early embryos. Recently, modulation of fusion/fission balance has been suggested to play a relevant role in the survival and maintenance of ESCs [6]. However, the overall mechanisms through which mitochondrial dynamics and biogenesis are controlled within PSCs remain to be fully elucidated.

When somatic cells are reprogrammed into iPSCs, they undergo extensive remodeling of genetic and epigenetic circuitry, which in turn may impact on mitochondrial properties. Initial studies with the mitochondrial dye MitoTracker Green showed that active mitochondria are mainly present within cells located at the borders of iPSC colonies, which are commonly considered to be on the verge of differentiation $[3,7]$. This suggested that undifferentiated PSCs may contain less active mitochondria (fig. 1). Ultrastructural investigations confirmed that iPSCs possess immature-appearing mitochondria, in a the features of mitochondria within somatic fibroblasts. Upper panel: mitochondria stained with dye MitoTracker Green, which indicates functionally-active mitochondria. In PSCs the dye mainly stains cells on the verge of differentiation at borders of colonies. White Bar $10 \mu \mathrm{m}$. Lower panel: transmission electron microscope (TEM) images of mitochondria. Black bar: $260 \mathrm{~nm}$. similar fashion to ESCs [7-10]. Compared to their elongated tubular-shaped, branched, and cristae-rich somatic cell counterparts, iPSC mitochondria appear spherical, with undeveloped cristae, and with a perinuclear distribution (fig. 1). Mitochondrial number and mass have also been found to be reduced in iPSCs compared to their parental somatic cells $[7,11]$. It is tempting to speculate that this reduction may be due to mitophagy-like events induced during the process of cell fate conversion. Accordingly, the autophagy stimulator rapamycin has been found to improve the efficiency of reprogramming [12]. However, it remains to be demonstrated whether the reprogramming-associated reduction of mitochondria is caused by mitophagy activation [13]. Moreover, the role of fusion/fission balance and mitochondrial dynamics during iPSC generation has yet to be explored.

Overall, the mitochondrial number, morphology, ultrastructure, and distribution in iPSCs are different from those of somatic cells and remarkably similar to those of ESCs. Importantly, these mitochondrial features are only 
transiently associated with the pluripotent state. Upon differentiation, mitochondria within iPSCs, as well as those in ESCs, are capable of acquiring the properties of mitochondria within somatic cells $[14,15]$. This is particularly evident when iPSCs are differentiated into fibroblast-like cells. These derived fibroblasts (DFs) exhibit increased mtDNA copy number paralleled by maturation of mitochondria into tubular, cristae-rich structures, resembling the situation observed in the original somatic fibroblasts [7] (fig. 1). These findings further demonstrate the plasticity of cells and associated organelles and as such prompted stem cell scientists to move from descriptive studies to the investigation of the mitochondrial properties of PSCs from a functional and metabolic perspective.

\section{Reconfiguration of Energy Metabolism}

PSCs exhibit an elevated rate of proliferation and distinct cell cycle features compared to somatic cells, including a shortened G1 phase and a prolonged S phase. Hence, a conspicuous part of cellular energy has to be invested in building the biomass needed for continuous cell growth and at the same time preserve the undifferentiated status. The maintenance of PSCs might be associated with elevated anabolic demands. The introduction of exogenous pluripotency-associated transcription factors leads to extensive modulation of chromatin state, gene expression, and signal transduction to eventually enable the dramatic conversion of somatic cells to iPSCs. Accordingly, ESCs depend on specific metabolic prerequisites which may be paralleled in iPSCs.

Cancer cells are a known example of how rapid cell proliferation necessitates a distinct metabolic configuration. Otto Warburg was the first to observe that proliferating tumor cells preferentially convert glucose to lactate even in the presence of oxygen, a phenomenon known as aerobic glycolysis. This glycolytic shift, however, was originally attributed to functional mitochondrial impairment. On the other hand, several studies demonstrated that mitochondria can still function correctly upon cancer transformation, while it is the central carbon metabolism that undergoes extensive restructuring. This is believed to occur in order to ensure the supply of cellular building blocks required to support the increasing biomass [16], by enhancing the energy flux through the glycolytic cascade, from which cellular anabolic pathways are branching out.

It is now becoming evident that PSCs may also exhibit a cancer-like metabolic profile and that cellular repro-

Mitochondrial Function in Pluripotent

Stem Cells and Cellular Reprogramming gramming may be associated with a glycolytic switch in a similar fashion as cancer transformation. Indeed, bioenergetic analysis has shown that in comparison to somatic cells, PSCs produce a high amount of lactate coupled with lower ATP generation and lower oxygen consumption rates $[7,9,10,17,18]$. This is in agreement with the structural data, as the cristae are the well-known sites for mitochondrial respiration. Therefore, while somatic cells display highly organized, cristae-rich, and respiratory-active mitochondria to fuel their energetic demands by OXPHOS, PSCs mainly oxidize glucose by cytosolic glycolysis to generate ATP. Therefore in PSCs, mitochondrial ultrastructure, number, and resting membrane potential $(\Delta \Psi)$ need to be distinctly divergent. In fact, $\Delta \Psi$ was found to be elevated in human and mouse PSCs and this hyperpolarization is proposed to be a sign of reduced ATP production and glycolysis-based energetic flux [10, 19]. Importantly, however, despite their immature appearance, mitochondria in PSCs are still capable of producing ATP from OXPHOS and consuming oxygen [17, 20]. In particular, their high $\Delta \Psi$ poises them to instantly face the increasing energetic demands of differentiation, as glycolytic ATP sustains the appropriate $\Delta \Psi$ needed for cell proliferation and viability, by hydrolysis via the mitochondrial ATP synthase after import from the cytosol.

The metabolic profile of PSCs can also be identified by a specific transcriptional signature. Genes regulating gluconeogenesis and the first and last steps of glycolysis are upregulated in PSCs compared to somatic fibroblasts, together with genes involved in the non-oxidative branch of the pentose phosphate pathway (PPP) $[9,20]$. This implies that PSCs are characterized by a gene expression program favoring glycolysis and the diversion of glycolytic intermediates into the PPP, which provides nucleotide precursors essential for anabolic growth. Accordingly, metabolite quantification confirmed the accumulation of glucose-6-phosphate, indicative of PPP activation, in PSCs compared to somatic fibroblasts [20].

Mechanistically, the glycolytic state of PSCs may be acquired through the inhibition of the transport of pyruvate into mitochondria (pyruvate shunting), which in turn results in reduced availability of pyruvate as a substrate for the TCA cycle and in augmented conversion of pyruvate into lactate in the cytosol. This pyruvate reshuffling in PSCs may occur as a consequence of increased pyruvate dehydrogenase kinases 1 (PDK1) $[9,20]$, which inhibits pyruvate dehydrogenase, thus limiting the pyruvate entry into the TCA cycle. Accordingly, a small molecule activator of PDK1 significantly enhances the efficiency of cellular reprogramming [21]. In addition, PSCs 
may also express high levels of pyruvate kinase isoform M2 [25], whose reduced catalytic activity leads to decreased conversion of phosphoenolpyruvate into pyruvate, further diverting the energy flux outside the mitochondria into the upstream glycolysis-related pathways.

The preference of glycolysis over OXPHOS is evident in PSCs grown under both normoxic (atmospheric oxygen concentration) and hypoxic $\left(1-5 \% \mathrm{O}_{2}\right)$ conditions. Independent of available oxygen, PSCs are therefore incapable of adopting OXPHOS-based metabolism, despite its 19-fold higher efficiency of ATP generation compared to glycolysis. A similar glycolytic profile has also been observed in MSCs, suggesting that glycolysisfocused metabolism may constitute a prerequisite for stemness [22]. Accordingly, mouse epidermal progenitor cells could maintain their proliferation and differentiation potential even upon complete genetic ablation of the ETC, implying that tissue stem cells may rely on glycolysis and be independent from mitochondrial respiration [23]. Furthermore, cell fate conversion can be more efficiently achieved when using somatic cells exhibiting an elevated glycolytic profile, such as for example keratinocytes [20].

In the in vivo situation, stem cells mostly reside in tissues deprived of oxygen, like the uterus (for ESCs) or the bone marrow (for HSCs). It remains elusive whether stem cells in vivo do not consume oxygen because they populate hypoxic niches or their glycolytic metabolism represents an intrinsic property of stem cells regardless of their environment. Finally, the intricate relationship between energy metabolism, proliferation, and stemness still needs to be fully clarified, as HSCs appear to exhibit glycolytic features even if they are mostly quiescent in their undifferentiated state [24]. Perhaps the glycolysis-oriented metabolic structuring of stem cells may be important to support their specific features of self-renewal and potency, regardless of their proliferative condition.

\section{Metabolic Reprogramming in iPSCs: Cellular Adaptation or Enabling Step?}

The functional relevance of the metabolic reconfiguration occurring during iPSC induction still needs to be clarified. Originally, the mitochondrial and metabolic remodeling was interpreted as a simple adaptation to the pluripotent state. However, recent data in mouse fibroblasts indicated that the increase in $\Delta \Psi$ and the upregulation of specific glycolytic genes may take place early in the reprogramming process, before genes involved in self-renewal and pluripotency are activated [10]. Our ongoing work confirms these findings and indicates that the transcriptional upregulation of $P D K 1$ can be detected in human fibroblasts already in the first 3 days of reprogramming [25], further implying that the regulation of energy metabolism may represent an initiating step of iPSC derivation.

The cellular transition during nuclear reprogramming might occur in a distinct temporal pattern of transcriptional events. The first wave may be characterized by cMYC and KLF4, which could drive the downregulation of fibroblast-associated protein expression and the upregulation of a cluster of genes regulating cell cycle, proliferation, and glycolytic metabolism [25]. The second wave, marked by OCT4 and SOX2-mediated repression of developmental genes and activation of ESC-associated genes, finally enables the substantial epigenetic restructuring to successfully complete the reprogramming process. Depending on the delivery method, transcription factor-mediated iPSC generation is initiated in $1-5 \%$ of induced cells but is not completed in most of them. This emphasizes how cellular reprogramming is still a poorly understood stochastic process and the underlying mechanisms involved in the induction and maturation steps are still in need of further dissection.

Taken together, such recent evidence appears to suggest that the 'metabolic stem cell program' may precede the 'pluripotency program' during cell fate conversion [25]. This implies that the reconfiguration of energy metabolism possibly represents an enabling step of cellular reprogramming, a barrier that somatic cells have to overcome in order to become able to sustain the proliferative and biosynthetic needs that are required to attain and maintain pluripotency.

\section{Maintenance of the Redox Balance}

Mitochondria, besides being the cellular powerhouse, represent the primary generators of toxic reactive oxygen species (ROS), which are common OXPHOS by-products. ROS can in turn lead to oxidative damage to DNA, proteins, or lipids and are thus counteracted by antioxidant enzymes. If this balanced redox homeostasis is perturbed, oxidative stress can occur, leading to cellular detrimental effects, a situation that likely occurs during the aging process and age-associated neurodegeneration.

PSCs are particularly sensitive to redox imbalance and have been found to display low levels of oxidatively modi- 
fied proteins, lipids, and DNA in the undifferentiated state $[7,11]$. To guarantee extended cell survival and proliferation capacity, PSCs acquire elevated stress defense mechanisms and a strictly restrained redox state. This is of critical importance for PSCs in order to ensure maximal safeguard from genome instability. Therefore, antioxidant enzymes (UCP2, SOD2) are elevated in PSCs to balance ROS through minimized substrate oxidation and respiratory coupling $[11,17]$. Accordingly, it has been suggested that the cellular metabolome of PSCs contains elevated amounts of unsaturated metabolites, which are highly susceptible to oxygenation and hydrogenation reactions. This allows PSCs to quickly respond to increased metabolic oxidation through the initiation of differentiation programs, a process dubbed as 'chemical plasticity' [26].

In the context of redox balance, the glycolytic metabolism of iPSCs may exhibit additional advantages. First, by decreasing mitochondrial respiration, it leads to the reduction of unwanted ROS generation. Secondly, by enhancing the flux through the oxidative branch of the PPP, it provides the reducing factor $\mathrm{NADPH}$, required for the activity of antioxidant enzymes [27]. Hence, the shift to glycolytic metabolism may not only be beneficial for cell proliferation but also for the regulation of redox balance. Indeed, PSCs depend on a constant and rapid supply of both energy in the form of ATP and reducing equivalents, such as NADPH. Glycolysis might then be instrumental for two reasons since it provides both biomass precursors and protection against oxidative stress. Hence, restriction of OXPHOS to minimal levels might constitute an additional advantage for stem cells through the reduction of ROS-mediated cellular damage.

\section{mtDNA Integrity}

Mitochondria contain their own genetic material, a circular compact $16-\mathrm{kb}$ genome encoding for 13 polypeptides of the OXPHOS subunits, 2 rRNAs and 22 tRNAs, which are needed for the intramitochondrial translation machinery. With the exception of complex II, which is entirely encoded by the nucleus, OXPHOS complexes are encoded by both nuclear and mitochondrial DNA. The proper functioning of the respiratory chain thus depends on the intricate coordination of two distinct genomes, requiring sensitive nucleus-to-mitochondria and retrograde signaling pathways. Mitochondrial genetics is fundamentally different from mendelian genetics, as mtDNA is transmitted through the maternal line and is present in multiple copies per cell. When almost all copies of

Mitochondrial Function in Pluripotent

Stem Cells and Cellular Reprogramming
mtDNA are identical, the state is defined as 'homoplasmy', while 'heteroplasmy' describes a cell with a mixture of two or more mtDNA genotypes.

Mutations in mtDNA occur frequently, given the close proximity to ROS generated by the ETC and despite the presence of mtDNA repair mechanisms [28]. Normal organelle turnover by mitophagy can keep the content of severely mutated mtDNAs at heteroplasmic levels below a pathogenetic threshold. Nonetheless, over time mtDNA mutations can accumulate during aging causing multiple cellular dysfunctions [29]. mtDNA mutations are also a frequent cause of rare genetic disorders, with an overall incidence around 1:5,000 individuals. In every case, being the mtDNA mutations spontaneous or inherited, significant OXPHOS defects develop when the proportion of $\mathrm{mtDNAs}$ carrying a pathogenic mutation exceeds a critical threshold, which depends upon the mutation, the cell type, and the patient's genetic background.

Despite the clinical implications associated with mtDNA alterations, a few studies have examined the integrity of mtDNA within PSCs. Recently, hESCs have been found to exhibit large-scale mtDNA deletions at different proportions even at low passage number, although a clear functional effect could not be observed [30]. These data indicate that although there are high defense mechanisms in place in PSCs to counteract oxidative stress and maintain the redox balance, the mitochondrial genome of PSCs may still undergo mutational events.

In accordance, we recently demonstrated using deep sequencing analyses that retroviral-derived human iPSCs may acquire single mtDNA point mutations during cellular reprogramming [20]. These mutations did not seem to target any specific gene and were mainly not diseaseassociated, although they could also occur at mtDNA haplotype-defining positions. Since mtDNA haplotypes have been linked with distinct gene expression patterns and differentiation potency in mouse ESCs [31], it is tempting to speculate that this reprogramming-associated random mtDNA rearrangement may contribute to the high degree of heterogeneity seen among various iPSC lines. Reassuringly, a recent study conducted on episomal plasmid-derived human iPSCs detected a much lower extent of mtDNA rearrangement [32]. This suggests that the reprogramming method may influence mtDNA integrity and that the use of viruses may elicit an elevated oxidative stress response, ultimately leading to a higher number of mtDNA modifications.

In summary, these recent findings imply that the state of the mitochondrial genome should always be assessed before employing iPSC lines for disease modeling or clin- 
Table 1. Comparison of mitochondrial-related features in somatic fibroblasts and PSCs

\begin{tabular}{|c|c|c|}
\hline Feature & Somatic fibroblasts & Pluripotent stem cells \\
\hline Number & High & Low \\
\hline Maturation & Well-organized, stacked cristae & Underdeveloped cristae \\
\hline Membrane potential $(\Delta \Psi)$ & Low basal polarization & Hyperpolarization \\
\hline OXPHOS & Main source of ATP & Low contribution to the total cellular ATP levels \\
\hline TCA cycle & $\begin{array}{l}\text { At high levels, fuels ETC with electrons } \\
\text { (NADH) }\end{array}$ & $\begin{array}{l}\text { Downregulated gene expression (measurement } \\
\text { of metabolites is needed) }\end{array}$ \\
\hline Pentose phosphate pathway (PPP) & Relatively less employed & Might be induced \\
\hline Aerobic preference & Normoxic & Hypoxic \\
\hline Metabolome & Saturated & Unsaturated \\
\hline Redox balance & May be perturbed & Tightly regulated \\
\hline Oxidative damage & Present & Reduced to very low levels \\
\hline mtDNA integrity & Mutations may rise over time & $\begin{array}{l}\text { Mutations may be present and induced by } \\
\text { reprogramming }\end{array}$ \\
\hline Mitophagy & Enables organelle homeostasis & Potentially increased during reprogramming \\
\hline
\end{tabular}

ical applications, in order to exclude the presence of de novo reprogramming-induced mtDNA mutations. On the other hand, like a double-edged sword, the rearrangement of mtDNA upon reprogramming opens a window for selection of preferable mtDNA genotypes in patient cell lines, thereby deriving mtDNA mutation-free iPSCs from patients affected by mtDNA disorders [33-35].

\section{iPSCs in Aging and Rejuvenation}

iPSCs are a remarkable tool to study complex diseases and mitochondrial biology in general and may also be a specific asset in the context of aging research. Indeed, the investigation of the pathways needed to be activated in order to convert somatic cells to embryo-like cells may be instrumental in understanding the aging process and identify possible measures to counteract it [36]. The key question to address is then whether reprogramming cell fate may not only 'turn back the developmental clock', as it is often said, but also 'rejuvenate' the features of aging.

iPSCs have been recently obtained from fibroblasts derived from elderly subjects. This has been accomplished by several research groups, demonstrating that the individual age does not per se represent a reprogramming road block [36]. Reprogrammed aged fibroblasts may however be more susceptible to develop karyotype abnormalities, possibly due to the impaired redox homeostasis that may be associated with aging [19]. Nonetheless, agederived iPSCs exhibit similar mitochondrial properties of 


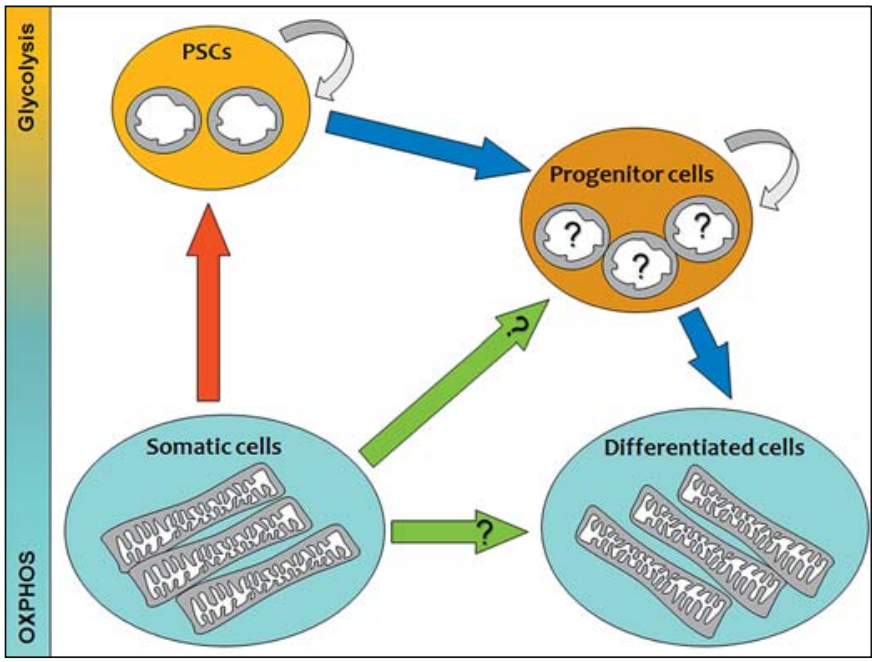

Fig. 2. Mitochondria in reprogramming and differentiation. Scheme depicting both known and unknown features of mitochondria during reprogramming and differentiation via intermediate progenitor cell population. Both PSCs and progenitor cells are capable of self-renewing for several passages (shown by white arrows). Red arrow indicates cellular reprogramming to pluripotency. Blue arrows indicate differentiation from PSCs. Green arrows designate direct reprogramming (or trans-differentiation) from somatic cells to proliferating progenitors or from somatic cells to differentiated cells of a different lineage. Mitochondria in somatic cells and in PSC-derived differentiated cells exhibit high number and mature tubular networks, while mitochondria within PSCs are mainly represented by few, round-shaped, immature organelles. The mitochondrial and metabolic features of progenitors cells obtained from PSCs still need to be fully characterized. Moreover, the properties of mitochondria within cells obtained through direct differentiation remain to be investigated.

iPSCs derived from young sources as well as hESCs. These include the round-shaped cristae-poor ultrastructure, the elevated $\Delta \Psi$, and the reduced level of ROS generation and oxidative DNA damage [19]. Moreover, aged-derived iPSCs are as sensible to drug-induced apoptosis as youngderived iPSCs, suggesting that the presence of karyotype aberrations may not necessarily predispose aged donor cells to cancer development [19]. On the other hand, the demonstration that reprogramming may induce mtDNA mutation events [20] may point out that cell fate conversion cannot be sufficient to erase the mtDNA alterations that accumulate over time upon aging.

Overall, it remains to be clarified whether all signs of aging can be erased during the conversion of aged cells into iPSCs. Nonetheless, the reprogramming technology may bring significant new knowledge for aging research and may provide the opportunity to model aging in a dish and identify measures to decelerate its development.

\section{Outlook}

Despite the well-known biological significance of mitochondria in cell functionality and the important implications in medical research, the role of mitochondria in the biology of stem cells has only been recently appreciated. The unique mitochondrial features of stem cells and reprogrammed cells are now starting to be described and understood (table 1). Importantly, mitochondrial structure and function have been even suggested as indicators of stem cell competence [37]. Despite this accumulating evidence, several questions remain to be addressed and more efforts are warranted to clearly dissect the functional role of mitochondria in PSCs, cellular reprogramming, and direct reprogramming (fig. 2). For example, it may be important to determine whether mitochondria within differentiated cells derived by direct reprogramming display similar characteristics to those obtained through the intermediate PSC state, which may rejuvenate or modulate key mitochondrial aspects. Also, the mitochondrial and metabolic properties of PSC-derived self-renewing progenitor cells still need to be fully characterized [38] (fig. 2). Overall, understanding the mitochondrial features of stem cells and their modulatory mechanisms may be critical in defying the properties of stem cells and may significantly improve aging research, disease modeling, and regenerative medicine applications.

\section{Acknowledgements}

We apologize to all the authors whose important works have not been cited due to space limitations. We thank Beatrix Fauler and Dr. Rudi Lurz for the TEM images. The authors declare no competing financial or commercial interests and acknowledge support from the Fritz Thyssen Foundation (grant AZ 10.11.2.160 to A.P.). J.A. acknowledges support from the German Federal Ministry of Education and Research (BMBF grant No. 01GN1005) and also BMBF grant No. 0315717A, a partner of the ERASysBio+ initiative supported under the EU ERA-NET Plus scheme in FP7.

References

Takahashi K, Yamanaka S: Induction of pluripotent stem cells from mouse embryonic and adult fibroblast cultures by defined factors. Cell 2006;126:663-676.

2 Sancho-Martinez I, Baek SH, Izpisua Belmonte JC: Lineage conversion methodologies meet the reprogramming toolbox. Nat Cell Biol 2012;14:892-899.

$\checkmark$ Cho YM, et al: Dynamic changes in mitochondrial biogenesis and antioxidant enzymes during the spontaneous differentiation of human embryonic stem cells. Biochem Biophys Res Commun 2006;348:1472-1478. 
4 St John JC, et al: The expression of mitochondrial DNA transcription factors during early cardiomyocyte in vitro differentiation from human embryonic stem cells. Cloning Stem Cells 2005;7:141-153.

5 Facucho-Oliveira JM, et al: Mitochondrial DNA replication during differentiation of murine embryonic stem cells. J Cell Sci 2007; 120(Pt 22):4025-4034.

6 6 Todd LR, et al: Growth factor erv1-like modulates Drp1 to preserve mitochondrial dynamics and function in mouse embryonic stem cells. Mol Biol Cell 2010;21:1225-1236.

7 Prigione A, et al: The senescence-related mitochondrial/oxidative stress pathway is repressed in human induced pluripotent stem cells. Stem Cells 2010;28:721-733.

8 Suhr ST, et al: Mitochondrial rejuvenation after induced pluripotency. PLoS One 2010; 5:e14095.

9 Varum S, et al: Energy metabolism in human pluripotent stem cells and their differentiated counterparts. PLoS One 2011;6:e20914.

10 Folmes CD, et al: Somatic oxidative bioenergetics transitions into pluripotency-dependent glycolysis to facilitate nuclear reprogramming. Cell Metab 2011;14:264-271.

11 Armstrong L, et al: Human induced pluripotent stem cell lines show stress defense mechanisms and mitochondrial regulation similar to those of human embryonic stem cells. Stem Cells 2010;28:661-673.

$\checkmark 12$ Chen T, et al: Rapamycin and other longevitypromoting compounds enhance the generation of mouse induced pluripotent stem cells. Aging Cell 2011;10:908-911.

$\checkmark 13$ Menendez JA, et al: mTOR-regulated senescence and autophagy during reprogramming of somatic cells to pluripotency: a roadmap from energy metabolism to stem cell renewal and aging. Cell Cycle 2011;10:3658-3677.

14 Prigione A, Adjaye J: Modulation of mitochondrial biogenesis and bioenergetic metabolism upon in vitro and in vivo differentiation of human ES and iPS cells. Int J Dev Biol 2010; 54:1729-1741.
15 Mandal S, et al: Mitochondrial function controls proliferation and early differentiation potential of embryonic stem cells. Stem Cells 2011;29:486-495.

16 Vander Heiden MG, Cantley LC, Thompson CB: Understanding the Warburg effect: the metabolic requirements of cell proliferation. Science 2009;324:1029-1033.

17 Zhang J, et al: UCP2 regulates energy metabolism and differentiation potential of human pluripotent stem cells. EMBO J 2011;30: 4860-4873.

18 Panopoulos AD, et al: The metabolome of induced pluripotent stem cells reveals metabolic changes occurring in somatic cell reprogramming. Cell Res 2011;22:168-177.

19 Prigione A, et al: Mitochondrial-associated cell death mechanisms are reset to an embryonic-like state in aged donor-derived iPS cells harboring chromosomal aberrations. PLoS One 2011;6:e27352.

20 Prigione A, et al: Human induced pluripotent stem cells harbor homoplasmic and heteroplasmic mitochondrial DNA mutations while maintaining human embryonic stem cell-like metabolic reprogramming. Stem Cells 2011; 29:1338-1348.

21 Zhu S, et al: Reprogramming of human primary somatic cells by OCT4 and chemical compounds. Cell Stem Cell 2010;7:651-655.

22 Chen CT, et al: Coordinated changes of mitochondrial biogenesis and antioxidant enzymes during osteogenic differentiation of human mesenchymal stem cells. Stem Cells 2008;26:960-968

23 Baris OR, et al: The mitochondrial electron transport chain is dispensable for proliferation and differentiation of epidermal progenitor cells. Stem Cells 2011;29:1459-1468.

-24 Suda T, Takubo K, Semenza GL: Metabolic regulation of hematopoietic stem cells in the hypoxic niche. Cell Stem Cell 2011;9:298-310.

25 Prigione A, et al: HIF1a modulates cell fate reprogramming through early glycolytic shift and up-regulation of PDK1-3 and PKM2. Stem Cells 2013, DOI: 10.1002/stem.1552.

-26 Yanes O, et al: Metabolic oxidation regulates embryonic stem cell differentiation. Nat Chem Biol 2010;6:411-417.
27 Ralser M, et al: Dynamic rerouting of the carbohydrate flux is key to counteracting oxidative stress. J Biol 2007;6:10.

28 Kazak L, Reyes A, Holt IJ: Minimizing the damage: repair pathways keep mitochondrial DNA intact. Nat Rev Mol Cell Biol 2012;13: 659-671.

-29 Prigione A, Cortopassi G: Mitochondrial DNA deletions induce the adenosine monophosphate-activated protein kinase energy stress pathway and result in decreased secretion of some proteins. Aging Cell 2007;6:619-630.

30 Van Haute L, et al: Human embryonic stem cells commonly display large mitochondrial DNA deletions. Nat Biotechnol 2013:31:20-23.

31 Kelly RD, et al: Mitochondrial DNA haplotypes define gene expression patterns in pluripotent and differentiating embryonic stem cells. Stem Cells 2012;31:703-716.

32 Cheng L, et al: Low incidence of DNA sequence variation in human induced pluripotent stem cells generated by nonintegrating plasmid expression. Cell Stem Cell 2012;10: 337-344.

33 Cherry AB, et al: Induced Pluripotent Stem Cells with a Pathological Mitochondrial DNA Deletion. Stem Cells 2013;31:1287-1297.

34 Fujikura J, et al: Induced pluripotent stem cells generated from diabetic patients with mitochondrial DNA A3243G mutation. Diabetologia 2012;55:1689-1698.

35 Folmes CD, et al: Disease-Causing Mitochondrial Heteroplasmy Segregated Within Induced Pluripotent Stem Cell Clones Derived from a Patient with MELAS. Stem Cells 2013; 31:1298-1308.

36 Mahmoudi S, Brunet A: Aging and reprogramming: a two-way street. Curr Opin Cell Biol 2012;24:744-756.

37 Parker GC, Acsadi G, Brenner CA: Mitochondria: determinants of stem cell fate? Stem Cells Dev 2009;18:803-806.

38 Birket MJ, et al: A reduction in ATP demand and mitochondrial activity with neural differentiation of human embryonic stem cells. J Cell Sci 2011;124(Pt 3):348-358. 